\title{
miR-374 mediates the malignant transformation of gastric cancer- associated mesenchymal stem cells in an experimental rat model
}

\author{
RUNBI JI ${ }^{1,2}$, XU ZHANG ${ }^{2}$, HUI QIAN ${ }^{2}$, HONGBING GU $^{1}$, ZIXUN SUN $^{2}$, FEI MAO $^{2}$, \\ YONGMIN YAN ${ }^{2}$, JINGYAN CHEN ${ }^{2}$, ZHAOFENG LIANG $^{2}$ and WENRONG XU ${ }^{2}$ \\ ${ }^{1}$ Department of Clinical Laboratory Medicine, The Affiliated People's Hospital of Jiangsu University, Zhenjiang, \\ Jiangsu 212002; ${ }^{2}$ Jiangsu Key Laboratory of Medical Science and Laboratory Medicine, \\ School of Medicine, Jiangsu University, Zhenjiang, Jiangsu 212013, P.R. China
}

Received January 3, 2017; Accepted June 30, 2017

DOI: 10.3892/or.2017.5831

\begin{abstract}
Mesenchymal stem cells (MSCs) are a critical component of the tumor microenvironment. Upon distinct pathological stimulus, MSCs show phenotypic and functional changes. Gastric cancer is one of the leading causes of cancer-related deaths worldwide. The roles and mechanisms of MSCs in gastric cancer have not been well characterized. In the present study, we investigated the roles of MSCs in the malignant transformation from gastritis to gastric cancer using an $N$-methyl- $N$ '-nitro- $N$-nitrosoguanidine (MNNG)induced gastric cancer model. We isolated MSCs from the gastric tissues of normal (RGN-MSCs) and MNNG-exposed rats (RGI-MSCs), and compared the biological properties of RGI-MSCs with RGN-MSCs. We found that RGI-MSCs had increased proliferative and migratory capabilities than these capacities noted in the RGN-MSCs. In addition, RGI-MSCs produced higher levels of IL-6, CXCL10 and MCP-1 than RGN-MSCs. Moreover, RGI-MSCs promoted the migration of normal gastric mucosa epithelial cells by inducing epithelial-mesenchymal transition (EMT). The upregulation of miR-374 in RGI-MSCs was partially responsible for their increased proliferative and migratory capabilities. Collectively, our findings provide new evidence for the roles of MSCs in gastric carcinogenesis, suggesting that targeting gastric cancer-associated MSCs may represent a novel avenue for gastric cancer therapy.
\end{abstract}

Correspondence to: Professor Wenrong Xu or Professor Xu Zhang, Jiangsu Key Laboratory of Medical Science and Laboratory Medicine, School of Medicine, Jiangsu University, 301 Xuefu Road, Zhenjiang, Jiangsu 212013, P.R. China

E-mail: icls@ujs.edu.cn

E-mail: xuzhang@ujs.edu.cn

Key words: mesenchymal stem cells, gastric cancer, microenvironment, $\mathrm{miR}-374$

\section{Introduction}

Mesenchymal stem cells (MSCs) are adult stem cells with self-renewal and multi-directional differentiation abilities. MSCs can be recruited to inflammatory and tumor sites by microenvironmental signals, where they undergo phenotypic and functional changes (1). For example, LPS-treated MSCs show increased expression of NO and IL-6 and decreased expression of TNF- $\alpha$ (2). MSCs pre-stimulated with IFN- $\gamma$ and TNF- $\alpha$ express a higher level of VEGF via the activation of the HIF-1 $\alpha$ signaling pathway, accelerating colon cancer growth and tumor angiogenesis in vivo (3). Although the plasticity of MSCs has been identified, the exact roles of MSCs in cancer and the mechanisms that mediate their response to microenvironmental signals have not been well studied.

Gastric cancer is the third most frequent cause of cancer-related death in China and the mortality rate for gastric carcinoma remains $>50 \%$ worldwide $(4,5)$. Normal gastric mucosa is subjected to multiple stages of malignant transformation, including different types of gastritis, varying degrees of atypical hyperplasia, finally evolving into gastric cancer. Cells at the early stage of carcinogenesis including the periods of simple and atypical hyperplasia, are reversible. Therefore, studies on the transformation from gastritis to cancer may have important implications for the control of gastric cancer.

Among all the established animal models of gastric cancer (6-8), the rat model induced with chemical carcinogens such as $N$-methyl- $N$ '-nitro- $N$-nitrosoguanidine (MNNG) is widely used due to the simple operation, cheap cost, easy keep and lower natural cancer rate (9). It has been shown that MNNG treatment results in the mutation of DNA and increased oxidative injury, which is critical to gastric carcinogenesis $(10,11)$. Furthermore, the various stages before gastric cancer in Wistar rats are similar to the tissue morphology of humans (12).

We previously reported the isolation of MSCs from human gastric cancer tissues and demonstrated that gastric cancerderived MSCs promote gastric cancer growth and metastasis. However, the mechanism responsible for the transition of MSCs in gastric cancer remains unknown. We hypothesized that the inflammatory microenvironment may induce changes 
in MSCs, promoting the transformation from gastritis to gastric cancer. To this end, we established a gastric cancer model in rats by the administration of MNNG in drinking water and a high-salt diet. MSCs were isolated from the gastric tissues of normal (RGN-MSCs) and MNNG-induced (RGI-MSCs) rats. We found that MSCs from the MNNG-induced rat gastric tissues had stronger abilities to proliferate and migrate. In addition, MSCs from the MNNG-induced rat gastric tissues produced higher levels of pro-inflammatory factors and showed more profound effects in promoting gastric mucosa epithelial cell migration. We further demonstrated that the increased expression of miR-374 may be associated with the phenotypic and functional changes in RGI-MSCs.

\section{Materials and methods}

Animal model. Four-week-old male Wistar rats (Laboratory Animal Center of Shanghai, Academy of Science, Shanghai, China) were kept at $23 \pm 3^{\circ} \mathrm{C}$ and $55 \pm 5 \%$ humidity. The control group was administered a normal diet and regular drinking water. The treatment group was fed with water containing $100 \mathrm{mg} / \mathrm{l}$ of MNNG [Tokyo Chemical Industry (TCI) Tokyo, Japan] for 40 weeks. A high-salt diet containing $8 \%$ sodium chloride was administered starting from the 5th week for 15 weeks. The body weight of each rat was weighed regularly every 4 weeks.

Histopathological staining. Gastric tissues were collected at different time points $(0,12,24,36$ and 48 weeks), processed in $4.0 \%$ paraformaldehyde, embedded with paraffin and sectioned into $4-\mu \mathrm{m}$ sections. The sections were subjected to hematoxylin and eosin (H\&E) staining and Alcian blue-periodic acid Schiff (AB-PAS) staining as previously described (13).

MSC isolation and cell culture. The fresh stomach tissues were cut into $1-\mathrm{mm}^{3}$ sized pieces and cultured in low-glucose Dulbecco's modified Eagle's medium (LG-DMEM) supplemented with $10 \%$ fetal bovine serum (FBS) (both from Life Technologies, Grand Island, NY, USA) and 1\% penicillin/streptomycin at $37^{\circ} \mathrm{C}$ in humid air with $5 \% \mathrm{CO}_{2}$. The medium was replaced every 3 days until adherent fibroblast-like cells appeared. Then, the cells were trypsinized and passaged into a new flask for further expansion. The cells in passage 3 were used for all the experiments. MSCs from normal gastric tissues were termed as RGN-MSCs and those from gastric tissues at 24 weeks after exposure to MNNG were termed as RGI-MSCs. The gastric mucosa epithelial cell line GES-1 (Institute of Biochemistry and Cell Biology at the Chinese Academy of Sciences, Shanghai, China) was cultured in RPMI-1640 medium (Life Technologies) with 10\% FBS. Cells were incubated at $37^{\circ} \mathrm{C}$ in a humidified cell culture incubator with $5 \% \mathrm{CO}_{2}$.

Osteogenic and adipogenic differentiation in vitro. RGN-MSCs and RGI-MSCs were seeded into 6-well plates at $1 \times 10^{5}$ cells/well and cultured in $10 \%$ FBS-containing DMEM with either osteogenic $(0.1 \mu \mathrm{M}$ dexamethasone, $10 \mu \mathrm{M}$ $\beta$-glycerophosphate, $50 \mu \mathrm{M}$ ascorbate-phosphate; Sigma, St. Louis, MO, USA) or adipogenic supplements (Cyagen,
Guangzhou, China). The medium was changed every 3 days and the cells were induced for 2 weeks. At the end of induction, the cells were subjected to neutrophil alkaline phosphatase (NAP) staining or Oil Red O staining.

Cell transfection. miRNA mimics and inhibitors were synthesized by GenePharma (Shanghai, China). RGN-MSCs and RGI-MSCs $\left(2 \times 10^{5}\right.$ cells/well) were plated in 6-well plate and transfected with miRNA mimics (5 nM), inhibitors (200 nM) or negative controls using Lipofectamine 2000 (Life Technologies).

Flow cytometry. RGN-MSCs and RGI-MSCs ( $1 \times 10^{6}$ cells) were trypsinized, washed twice in phosphate-buffered saline (PBS), and stained for $30 \mathrm{~min}$ on ice in fluorescein isothiocyanate (FITC)-conjugated or phycoerythrin (PE)-conjugated antibodies: CD29, CD44H, CD45 and CD90 (Becton-Dickinson, San Jose, CA, USA). Mouse PE-IgG and FITC-IgG were used as the isotype control.

Genetics and DNA contents. RGN-MSCs and RGI-MSCs were incubated with colchicine for $4 \mathrm{~h}$ during the logarithmic growth phase. The cells were collected, treated with $\mathrm{KCl}(0.075 \mathrm{M})$ for $30 \mathrm{~min}$, and fixed in methanol/acetic acid (1:1 v/v). Cell smears were subjected to Giemsa staining for chromosome analysis. For DNA content analysis, the cells were harvested, washed with PBS twice, and stained with $10 \mu \mathrm{g} / \mathrm{ml}$ propidium iodide (Sigma) in $500 \mu \mathrm{l}$ PBS (containing $100 \mu \mathrm{g} / \mathrm{ml}$ RNase) for $30 \mathrm{~min}$ in the dark at room temperature. The distribution of cells at different phases of the cell cycle was analyzed using a flow cytometer (FACSCalibur; BD Biosciences, Franklin Lakes, NJ, USA).

Cell growth curve and cell colony formation. RGN-MSCs and RGI-MSCs were seeded into 24 -well plates $\left(5 \times 10^{3}\right.$ cells/well). The cell numbers were counted for each group at indicated time points. RGN-MSCs and RGI-MSCs were resuspended in 6 -well plates $\left(1 \times 10^{5}\right.$ cells/well) and incubated for 14 days. Colonies were fixed with methanol, stained with crystal violet and counted. For miRNA transfection, the cells were collected at $24 \mathrm{~h}$ after transfection and seeded in the 24-well plates ( $2 \times 10^{5}$ cells/well). All the experiments were carried out in triplicate.

Western blotting. Cells were lysed in RIPA buffer supplemented with proteinase inhibitors. A total of $200 \mu \mathrm{g}$ proteins was loaded and separated using $10 \%$ sodium dodecyl sulfate-polyacrylamide gel electrophoresis (SDS-PAGE). The proteins were transferred to a polyvinylidene difluoride (PVDF) membrane, blocked in 5\% (w/v) non-fat milk and incubated with the primary antibodies at $4^{\circ} \mathrm{C}$ overnight. The sources of primary antibodies were as follows: anti-Oct4, antiSox2, anti-Lin28B, anti-Kif4, anti-vimentin (Cell Signaling Technology, Danvers, MA, USA), anti-E-cadherin and anti-Ncadherin (Santa Cruz Biotechnology, Santa Cruz, CA, USA), anti-GAPDH (Kangcheng Biotechnology, Shanghai, China). After incubation with the secondary antibodies (Kangcheng Biotechnology) at $37^{\circ} \mathrm{C}$ for $1 \mathrm{~h}$, the signals were visualized using a chemiluminescent imaging system (Beijing Sage Creation Science, Beijing, China). 
A

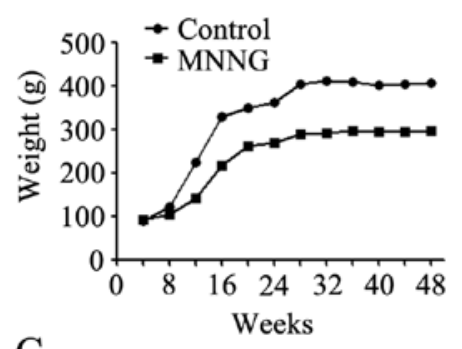

C
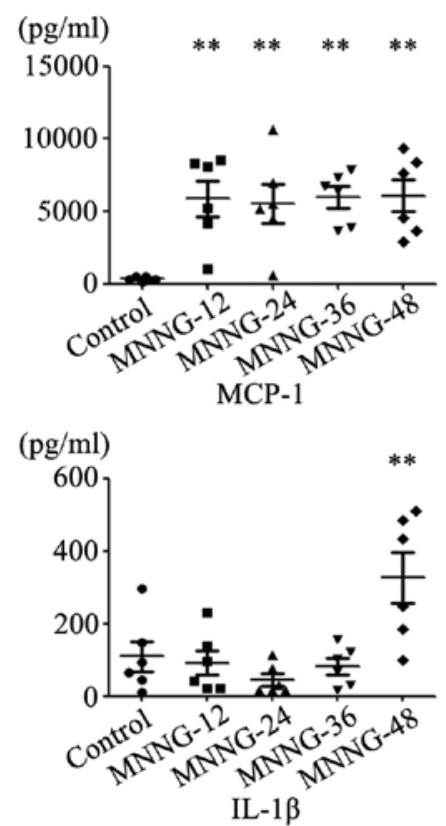

B

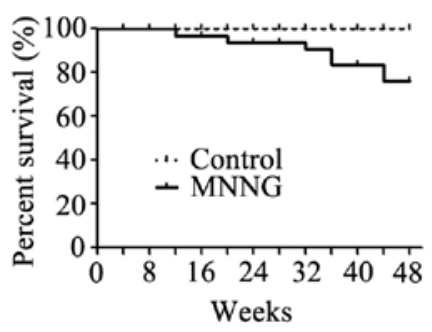

$(\mathrm{pg} / \mathrm{ml})$

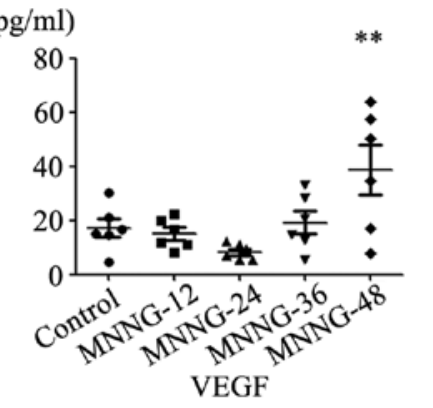

$(\mathrm{pg} / \mathrm{ml})$

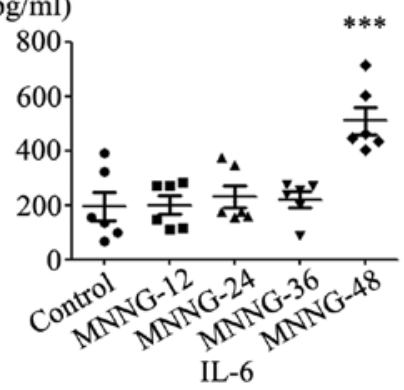

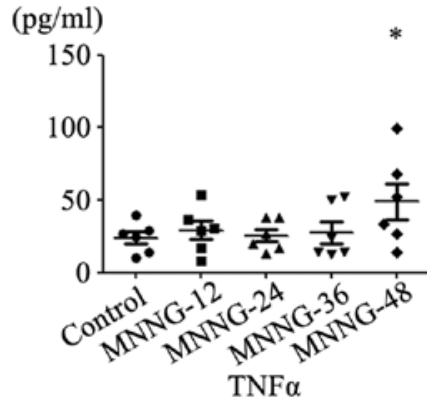

$\mathrm{TNF} \alpha$

Figure 1. (A) The body curves of rats in the control and MNNG groups. (B) The survival curves of rats in the control and MNNG groups. (C) Expression of pro-inflammatory factors in the serum of rats as detected using Luminex assay; ${ }^{*} \mathrm{P}<0.05 ;{ }^{* *} \mathrm{P}<0.01 ;{ }^{* * *} \mathrm{P}<0.001$.

Transwell migration assay. For RGN-MSCs and RGI-MSCs, cells $\left(5 \times 10^{4}\right.$ cells/well) were plated into the top chamber of Transwell plates and medium containing 10\% FBS was placed into the bottom chamber of Transwell plates $(8-\mu \mathrm{m}$ pore size; Corning Inc., Corning, NY, USA). For GES-1, cells $\left(5 \times 10^{4}\right.$ cells/well) were plated into the top chamber of Transwell plates and the supernatant of RGN-MSCs or RGI-MSCs was placed in the bottom chamber. After incubation at $37^{\circ} \mathrm{C}$ in $5 \%$ $\mathrm{CO}_{2}$ for $10 \mathrm{~h}$, the cells remaining on the upper surface of the membrane were removed with a cotton swab. Cells on the lower surface of the membrane were fixed and stained with crystal violet. The migratory ability of the cells was determined by counting the cells in at least 6 fields for each assay.

Luminex assay. The serum of rats in the control and the treatment group were collected at different time points $(0,12$, 24,36 and 48 weeks). The supernatant from the RGN-MSCs and RGI-MSCs was also collected. The Rat Cytokine and Chemokine Magnetic Bead Panel kit (cat.\#RECYTMAG-65K; Merck Millipore, Darmstadt, Germany) was designed to detect granulocyte macrophage colony-stimulating factor (GM-CSF), granulocyte colony stimulating factor (G-CSF), IL-10, IL-6, IL-4, IL-1 $\beta$, monocyte chemoattractant protein-1 (MCP-1), tumor necrosis factor- $\alpha$ (TNF- $\alpha$ ) and vascular endothelial growth factor (VEGF). All procedures were processed according to the manufacturer's instructions. The signal was detected and analyzed using the Luminex 200 System (Merck Millipore).

RNA isolation and reverse transcription-quantitative PCR. Total RNA was isolated from the cells using TRIzol reagent (Invitrogen, Carlsbad, CA, USA). Serum miRNAs were extracted from $400 \mu \mathrm{l}$ of serum using the miRNeasy Mini kit and reversely transcribed using the miScript II RT kit (both from Qiagen, Hilden, Germany). The levels of miRNAs were detected using the miScript SYBR-Green PCR kit (Qiagen) in a Bio-Rad fluorescence thermal cycler (Bio-Rad, Hercules, CA, USA). The relative expression levels of the miRNAs were normalized to that of U6.

Statistical analyses. All data are expressed as mean \pm SD. SPSS software was used to analyze all the data (SPSS, Inc., Chicago, IL, USA). The means of different treatment groups were compared by two-way ANOVA, LSD-t test. A P-value $<0.05$ was considered as statistically significant.

\section{Results}

$M N N G$ exposure increases the expression of inflammatory factors in rats. The weight of rats in the control group increased rapidly from the 4 th week, and became stabilized in the 24th week. The rats in the MNNG treatment group were 
A

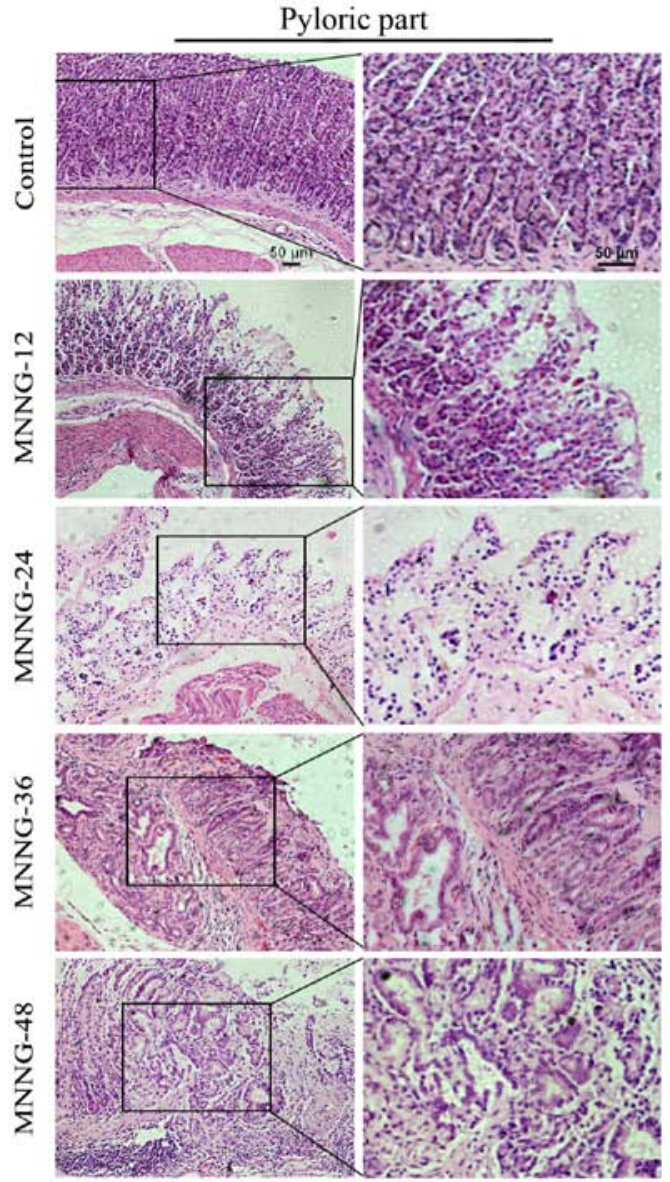

B

D

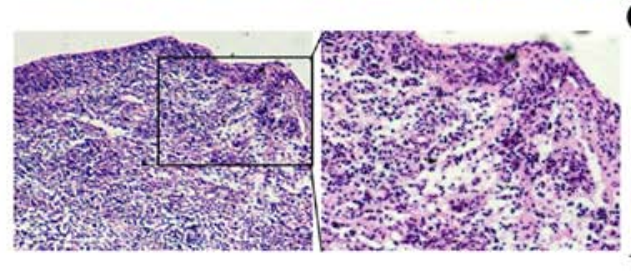

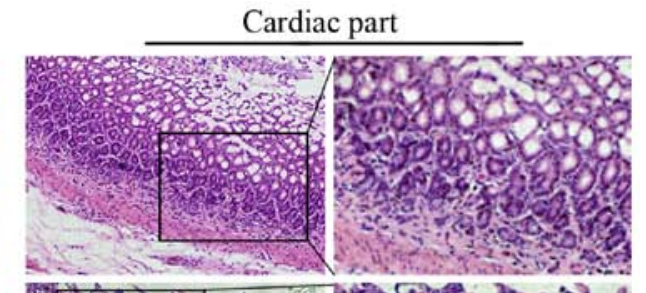
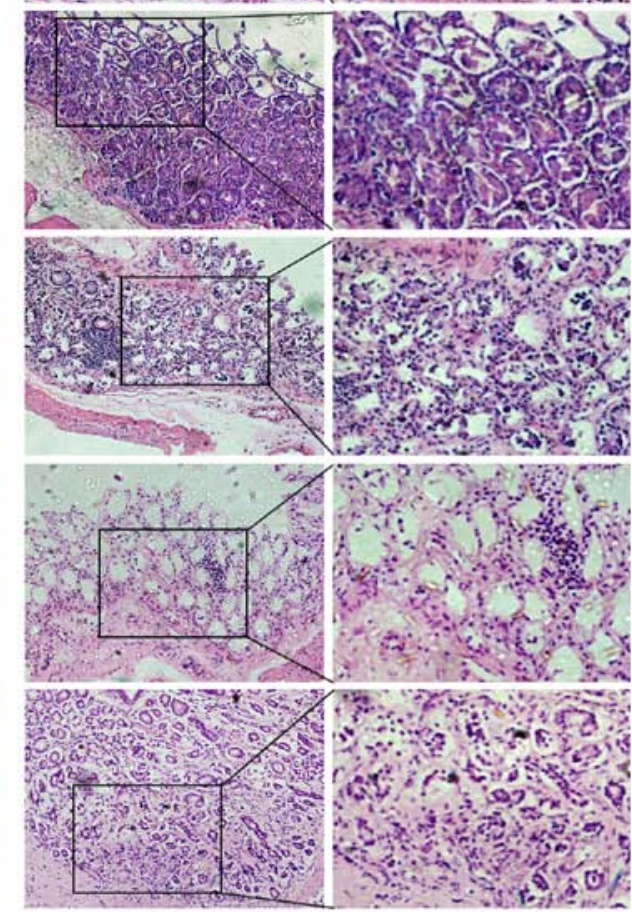

$\mathrm{C}$
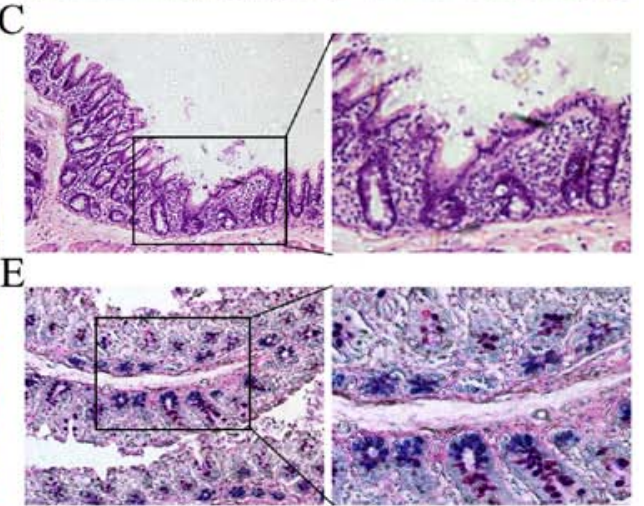

Figure 2. Histology of the control and MNNG-induced rat gastric tissues. (A) Superficial gastritis was noted in the MNNG-12 group, atrophic gastritis in the MNNG-24 group and the cardiac part of the MNNG-36 group, and atypical hyperplasia in the pyloric part of the MNNG-36 and MNNG-48 groups. (B) H\&E staining of ulcers in the MNNG-36 group. (C) H\&E staining of gastric tissues in the MNNG-36 group. Intestinal metaphase changes were observed. Goblet cells were noted in the gastric mucosa. (D) H\&E staining for gastric tissuse in the MNNG-48 group. Esophageal-like sarcoma lesions were observed. (E) Small intestinal type metaplasia was detected using AB-PAS staining. MNNG-12, 24, 36 and 48 represent the rats treated with MNNG for $12,24,36$ and 48 weeks, respectively. Original magnification of the left panel, x100; the right panel, x200; scale bar, $50 \mu \mathrm{m}$.

lighter than the normal rats at all time periods. The average weight of the normal adult male Wistar rats was $400 \mathrm{~g}$, while the average weight of rats in the MNNG treatment group was only $300 \mathrm{~g}$ at the 48th week (Fig. 1A). Some rats in the MNNG treatment group could not tolerate the exposure to MNNG and started to die at the 24th week after MNNG treatment. The survival rate in the MNNG treatment group was $\sim 75 \%$ at the end of the experiment (Fig. 1B).

We then detected the expression of inflammatory factors including GM-CSF, IL-1 $\beta$, IL-4, IL-6, IL-10, MCP-1, TNF- $\alpha$ and VEGF in the serum of rats using Luminex assay. The expression of GM-CSF, IL-4 and IL-10 was undetectable in the serum of rats. The expression level of MCP-1 in the serum of the MNNG-treated rats increased notably at the 12th week after MNNG exposure. However, there was no further change in the expression level of MCP-1 over time. The expression levels of VEGF, TNF- $\alpha$, IL-1 $\beta$ and IL- 6 in the serum of the MNNG-treated rats were higher than those in control group at the 48th week after MNNG exposure (Fig. 1C).

Establishment of the MNNG-induced gastric cancer model in rats. As shown in Fig. 2A, the structure of the gastric mucosa of 
A
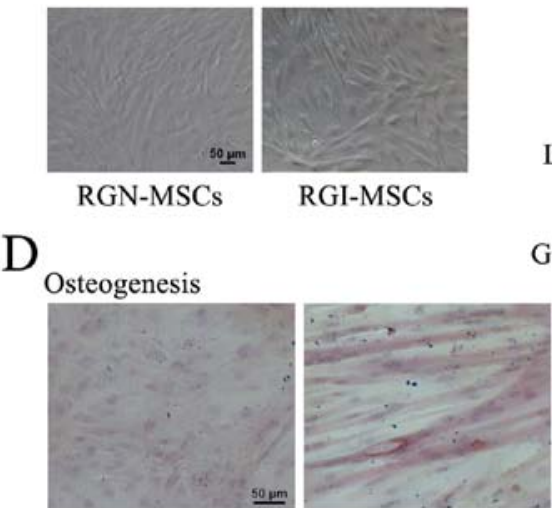

Adipogenesis

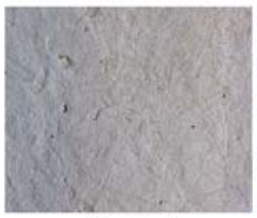

Control

E

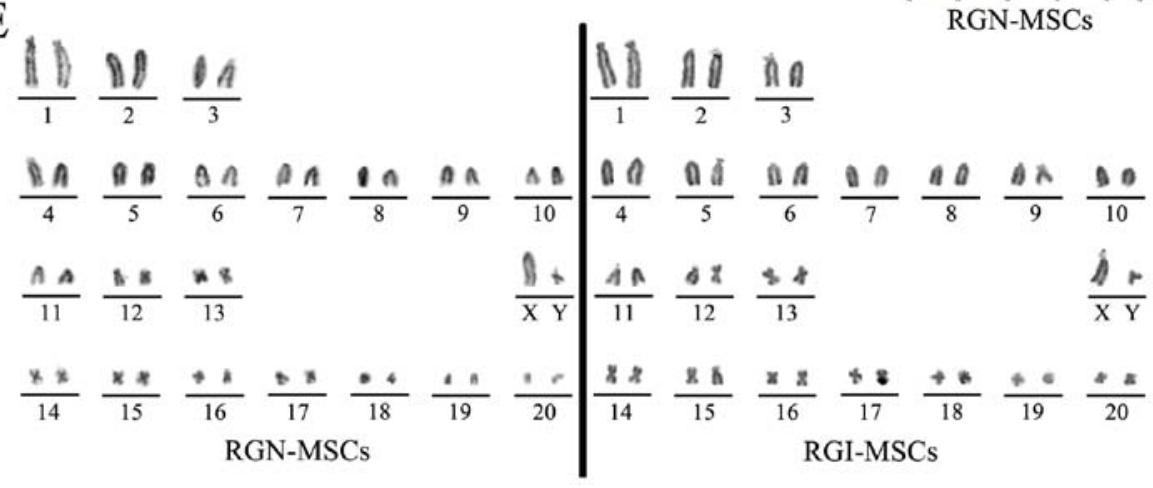

B

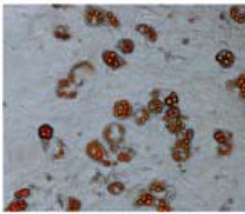

RGN-MSCs

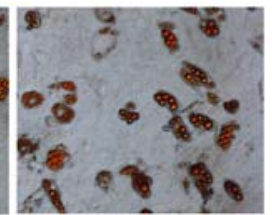

RGI-MSCs
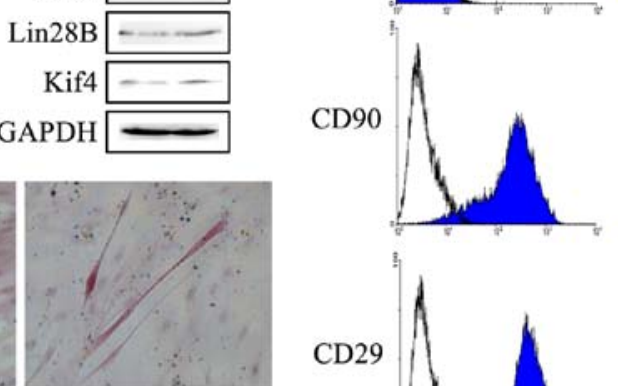

GAPDH

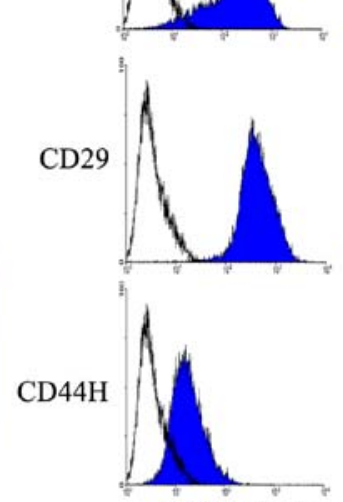

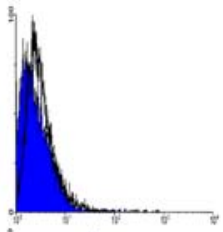

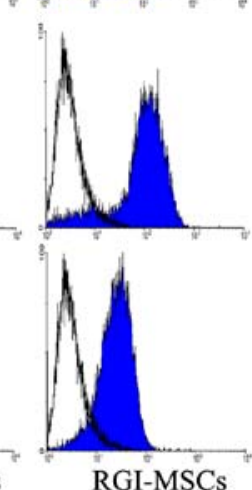

RGI-MSCs

Figure 3. Characterization of MSCs from the gastric tissues of normal and MNNG-induced rats. (A) Morphology of MSCs from the gastric tissues of normal (RGN-MSCs) and MNNG-induced (RGI-MSCs) rats. Original magnification, x100; scale bar, $50 \mu \mathrm{m}$. (B) Expression of stem cell factors including Oct4, Sox2, Lin28B, K1f4 in RGN-MSCs and RGI-MSCs was determined using western blotting. (C) Expression of CD29, CD44H, CD90 and CD34 in RGNMSCs and RGI-MSCs was determined using flow cytometry. (D) Osteogenic and adipogenic differentiation abilities of RGN-MSCs and RGI-MSCs. Original magnification, $\mathrm{x} 200$; scale bar, $50 \mu \mathrm{m}$. (E) The karyotypes of RGN-MSCs and RGI-MSCs.

the normal rats was complete. The boundaries of the glandular and glandular epithelium were clear. The dense glands had uniform size and the shape of the glandular lumen was regular. At 12 weeks after MNNG exposure, the mucosal layer of the gastric tissues became thinner and displayed focal or diffuse lesions, which manifested as superficial gastritis (Fig. 2A). The gastric mucosa of the rats treated with MNNG for 24 weeks became even thinner with visible inflammatory cell infiltration. The size of the glands became smaller and the number of the glands was reduced. The normal gastric pit became shallower, which could be diagnosed as atrophic gastritis (Fig. 2A). The inner gastric mucosa of rats treated with MNNG for 36 weeks had fibroid tissue. There was inflammatory granuloma or ulcer-like pathology, namely the surface was covered by inflammatory exudates (white blood cells, cellulose), under which were necrotic tissue layer, granulation tissue layers and layers of old scar tissue. The glands were shrinking with vacuole goblet cells. Therefore, the pathological changes at this stage were a mixture of atrophic gastritis (Fig. 2A), ulcers (Fig. 2B), and intestinal metaphase (Fig. 2C). After exposure to MNNG for 48 weeks, the glandular structure disappeared and showed disorders with irregular shape. There were a large number of inflammatory cells with disorganized size surrounding the basement membrane, which was judged as atypical hyperplasia (Fig. 2A). The cardiac part of the cauliflower uplift was identified to have benign esophageallike sarcoma lesions (Fig. 2D). Normal gastric epithelial cells secrete neutral mucous and are positive for periodic acid-Schiff (PAS) reaction (shown in red). Small intestinal epithelial cells secrete acid mucus and are positive for alcian blue reaction (shown in blue). The mucous mixed with neutral and acidic mucous are shown in purple. To determine the subtype of gastric intestinal metaplasia, the stomach tissue sections were used for Alcian blue-Schiff (AB-PAS) staining. The mucous glands were mixed with blue and purple nuclei, indicating that the subtype of intestinal metaplasia was small intestinal type (Fig. 2E). The incidence of atrophic gastritis, atypical hyperplasia, appaloosa and adenocarcinoma was 100 , $85.4,14.6$ and $0 \%$, respectively at 48 weeks in the MNNG treatment group. 


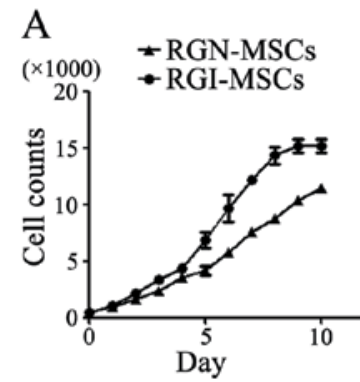

$\mathrm{C}$

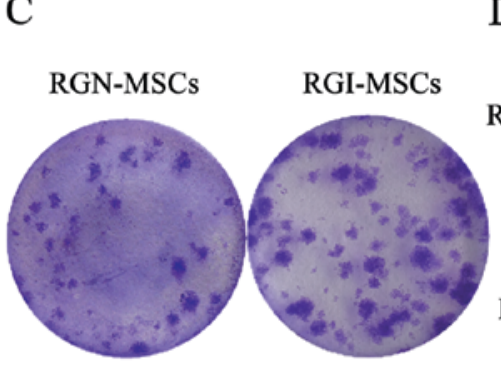

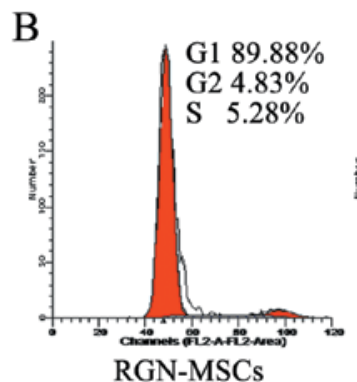
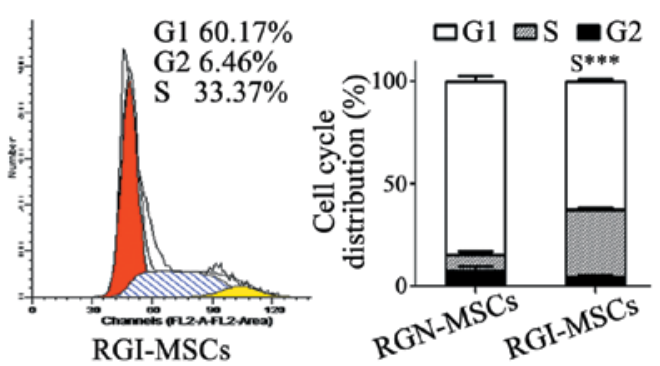

$\mathrm{D}$
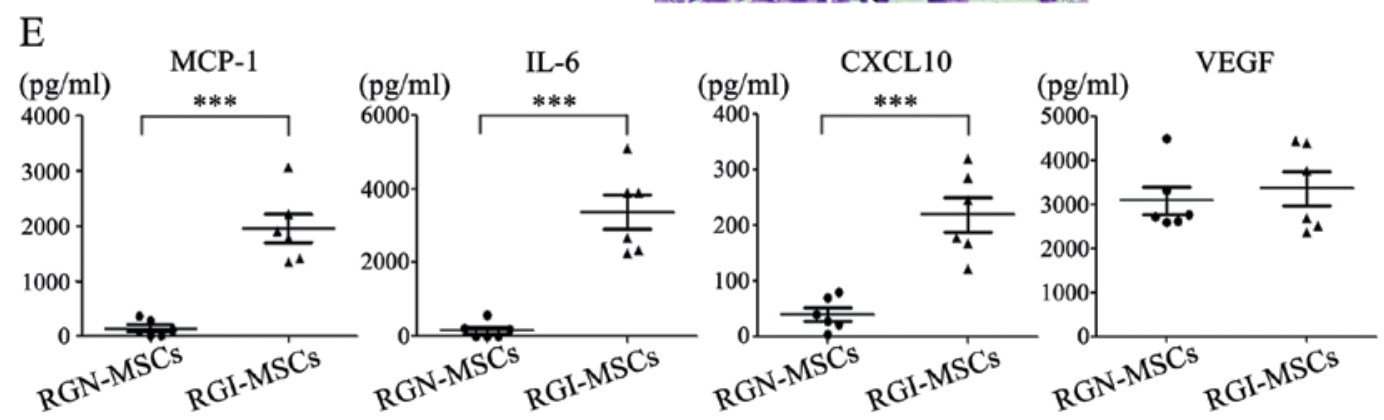

Figure 4. Comparison of the biological properties of RGI-MSCs and RGN-MSCs. (A) Growth curves of RGN-MSCs and RGI-MSCs. (B) Cell cycle distribution of RGN-MSCs and RGI-MSCs was determined using flow cytometry; ${ }^{* * *} \mathrm{P}<0.001$. (C) Cell colony formation assays for RGN-MSCs and RGIMSCs. (D) Transwell migration assays for RGN-MSCs and RGI-MSCs. Original magnification of the left panel, x100; the right panel, x200; scale bar, $50 \mu \mathrm{m}$; ${ }^{* * * *} \mathrm{P}<0.001$. (E) The production of pro-inflammatory factors in RGN-MSCs and RGI-MSCs was determined using Luminex assay; ${ }^{* * * *} \mathrm{P}<0.001$.

Isolation and characterization of MSCs from the gastric tissues of normal and $M N N G$-treated rats. After primary culture for 14 days, RGN-MSCs and RGI-MSCs adhered to the plastic surface of the culture plates. These cells began to form colonies and appeared like long spindle-shaped fibroblastic cells in the initial plating for $\sim 20$ to 30 days (Fig. 3A). The results of western blotting showed that both RGN-MSCs and RGI-MSCs expressed stem cell markers including Oct4, Sox2, Lin28B, and Klf4 (Fig. 3B). RGN-MSCs and RGI-MSCs were positive for $\mathrm{CD} 25, \mathrm{CD} 44 \mathrm{H}$ and $\mathrm{CD} 90$, but negative for CD45 (Fig. 3C). RGN-MSCs and RGI-MSCs could be induced to differentiate into osteocytes and adipocytes at 2 weeks after induction (Fig. 3D). The karyotypes of the RGN-MSCs and RGI-MSCs were normal, with 20 pairs of autosomes and one pair of sex chromosomes. No deletions or translocations in chromosomes were detected (Fig. 3E).

RGI-MSCs show increased proliferative and migratory abilities and produce higher levels of pro-inflammatory factors than RGN-MSCs. The results of the cell growth curves showed that the RGI-MSCs proliferated more quickly than that noted for the RGN-MSCs. The number of RGN-MSCs was $\sim 3 / 4$ that of the RGI-MSCs at 10 days of culture (Fig. 4A). The results of cell cycle analysis showed that the percentage of RGI-MSCs at the S phage $(32.8 \pm 1.39 \%)$ was higher than that of the RGN-MSCs $(7.75 \pm 2.23 \%)$ (Fig. 4B). The number of cell colonies formed by RGI-MSCs was significantly higher than that of the RGN-MSCs. The average diameter of the cell colonies formed by the RGI-MSCs was larger than that of the RGN-MSCs after culture for 14 days (Fig. 4C). In the cell Transwell migration assay, the number of migrated RGI-MSCs was 2-fold higher than that of the RGN-MSCs (Fig. 4D). To analyze the cytokine profiles of RGN-MSCs and RGI-MSCs, we performed Luminex assay to determine the levels of several pro-inflammation factors, including G-SCF, IL-10, IL-6, IL-1 $\beta$, MCP-1, TNF- $\alpha$ and VEGF, in the supernatant of the RGN-MSCs and RGI-MSCs. We observed that the levels of IL-6, CXCL-10 and MCP-1 were markedly upregulated in the supernatant of the RGI-MSCs. The level of VEGF had no significant difference between the RGN-MSCs and RGI-MSCs (Fig. 4E). The expression of other factors (G-CSF, IL-10, IL-1 $\beta$ and TNF- $\alpha$ ) were undetectable in both the RGN-MSCs and RGI-MSCs.

RGI-MSCs promote the migration of gastric mucosa epithelial cells more profoundly than RGN-MSCs. The gastric mucosal epithelial cells were treated with the supernatant of RGN-MSCs and RGI-MSCs and the migratory abilities of the 
A
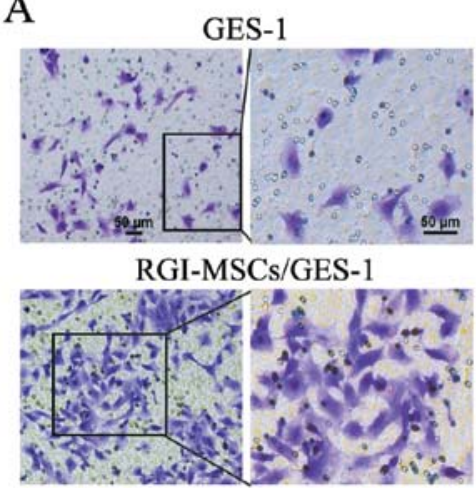

RGN-MSCs/GES-1
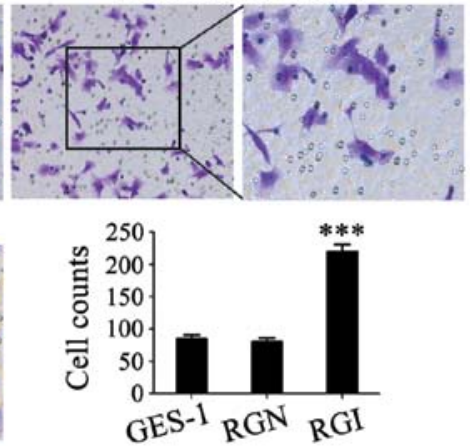

B

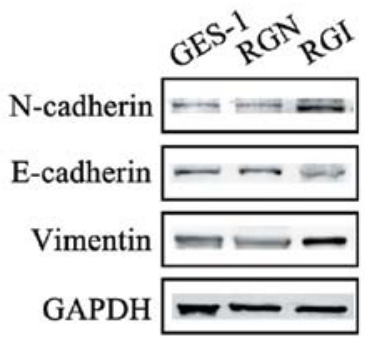

Figure 5. Effects of RGI-MSCs and RGN-MSCs on gastric mucosal epithelial cells migration and EMT. (A) RGI-MSCs promoted the migration of gastric mucosal epithelial cells more profoundly than RGN-MSCs. Original magnification of the left panel, $\mathrm{x} 100$; the right panel, $\mathrm{x} 200$; scale bar, $50 \mu \mathrm{m}$; ${ }^{* * * *} \mathrm{P}<0.001$. (B) Expression of E-cadherin, $\mathrm{N}$-cadherin and vimentin in gastric mucosal epithelial cells after treatment with the supernatant from RGI-MSCs and RGN-MSCs was determined using western blotting.
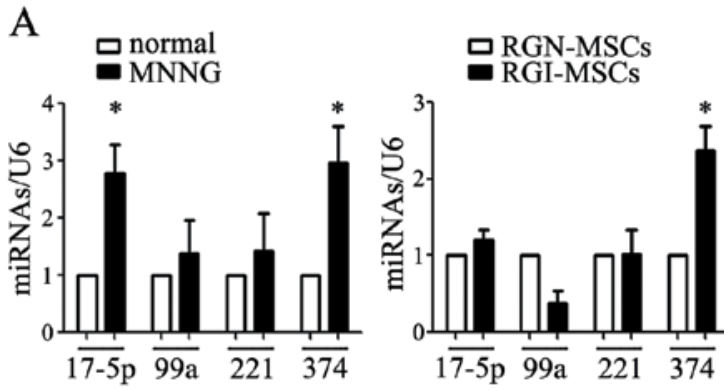

$\mathrm{B} \quad \mathrm{C}$
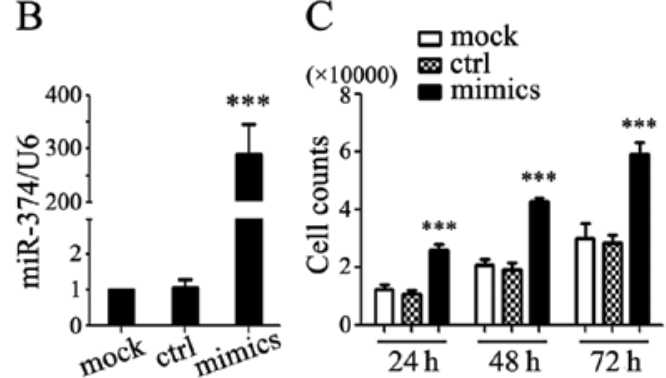

$\mathrm{D}$
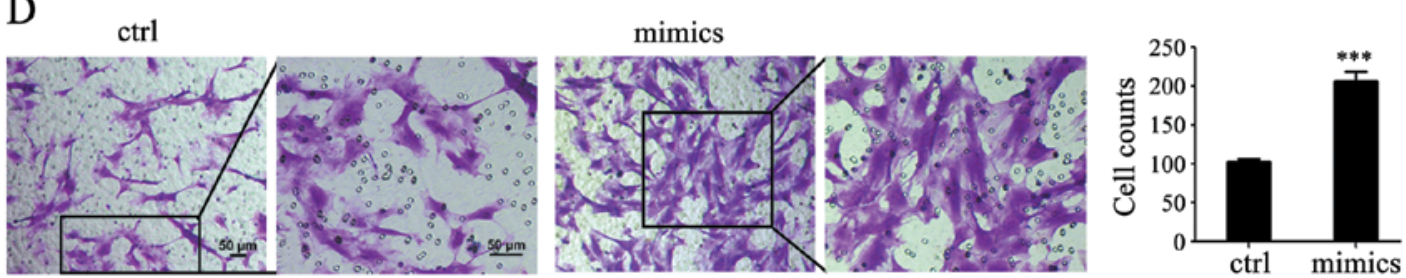

$\mathrm{E}$

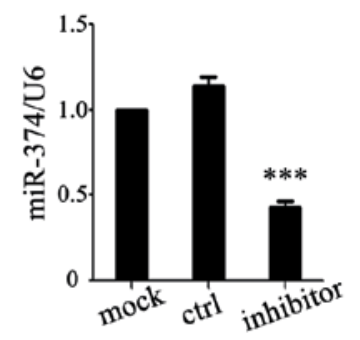

G $\quad$ ctrl

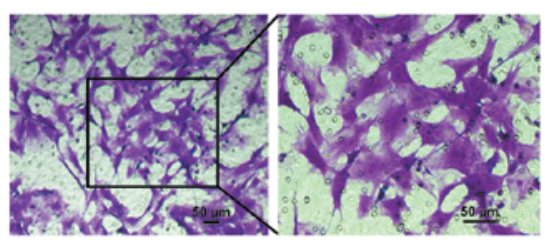

$\mathrm{F}_{(\times 10000)}$ 口 mock

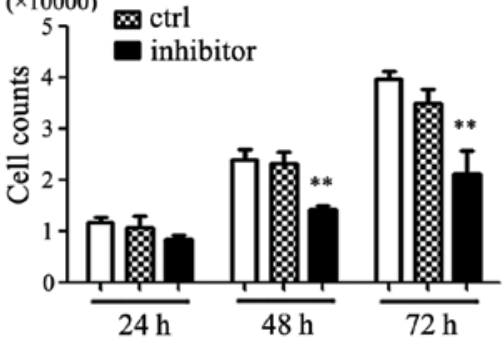

inhibitor

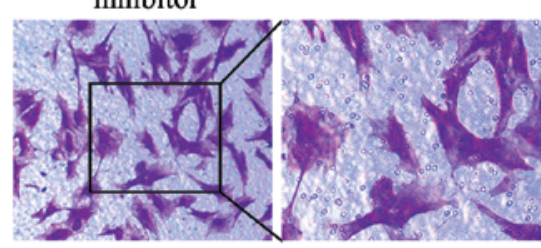

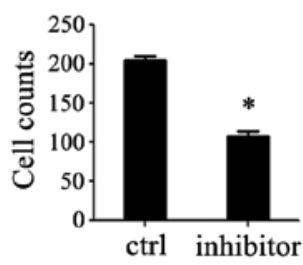

Figure 6. miR-374 is overexpressed in RGI-MSCs and mediates their increased proliferation and migration abilities. (A) The expression of miRNAs (miR17-5p, miR-99a, miR-221 and miR-374) in the serum of normal and MNNG-induced rats and in RGN-MSCs and RGI-MSCs; "P<0.05. (B) The expression of miR-374 in RGN-MSCs transfected with control and miR-374 mimics; ${ }^{* * *} \mathrm{P}<0.001$. (C) Cell counting assays for RGN-MSCs transfected with control and miR-374 mimics at the indicated time points; ${ }^{* * *} \mathrm{P}<0.001$. (D) Transwell migration assays for RGN-MSCs transfected with control and miR-374 mimics. Original magnification of the left panel, x100; the right panel, $\mathrm{x} 200$; scale bar, $50 \mu \mathrm{m} ;{ }^{* * *} \mathrm{P}<0.001$. (E) Expression of miR-374 in RGI-MSCs with control and miR-374 inhibitor; ${ }^{* * *} \mathrm{P}<0.001$. (F) Cell counting assays for RGI-MSCs transfected with control and miR-374 inhibitor at the indicated time points; ${ }^{* *} \mathrm{P}<0.01$. (G) Transwell migration assays for RGI-MSCs transfected with control and miR-374 inhibitor. Original magnification of the left panel, $\mathrm{x} 100$; the right panel, $\mathrm{x} 200$; scale bar, $50 \mu \mathrm{m} ;{ }^{*} \mathrm{P}<0.05$. 
treated cells were determined using Transwell migration assay. The number of GES-1 cells migrating through the Transwell membrane was higher than that in the control group $(\sim 2.5-$ fold increase). Whereas, there was only a minimal increase in the number of migrated GES-1 cells in the RGN-MSC group compared that in the control group (Fig. 5A). The results of western blotting showed that the expression of E-cadherin expression decreased while that of $\mathrm{N}$-cadherin and vimentin increased after treatment with the supernatant of the RGI-MSCs for $48 \mathrm{~h}$ (Fig. 5B). No significant changes in the expression of E-cadherin, $\mathrm{N}$-cadherin and vimentin were observed in GES-1 cells treated with the supernatant of the RGN-MSCs.

miR-374 is upregulated in RGI-MSCs and is responsible for the increased proliferative and migratory abilities of RGI-MSCs. In our preliminary study, we used an Exiqon miRCURY LNA Array to evaluate the expression profile of miRNAs between MSCs from human gastric cancer and non-cancerous gastric tissues. We identified 177 upregulated and 160 downregulated miRNAs, in which the expression of miR-17-5p, miR-99a, miR-221 and miR-374 was differentially significant (15). We performed qRT-PCR to determine the expression of several microRNAs in the serum of normal and MNNG-induced rats. As shown in Fig. 6A, the expression levels of miR-17-5p and miR-374 were significantly upregulated in the serum of MNNG-induced rats compared to that in normal rats. The expression levels of miR-99a and miR-221 had no significant differences between MNNG-induced rats and normal rats. We also detected the expression levels of these miRNAs in the RGN-MSCs and RGI-MSCs. We found that only the expression level of miR-374 was notably increased in the RGI-MSCs compared to that in the RGN-MSCs (Fig. 6A). We overexpressed miR-374 in the RGN-MSCs and found that miR-347-transfected RGN-MSCs proliferated more quickly than the cells transfected with the control mimics (Fig. 6B and C). miR-374 overexpression also significantly promoted the migration of RGN-MSCs (Fig. 6D). We inhibited miR-374 expression in the RGI-MSCs using miRNA inhibitors and found that the inhibition of miR-374 retarded the proliferation of RGI-MSCs at 48 and $72 \mathrm{~h}$ after transfection (Fig. 6E and F). The number of migrated RGI-MSCs was also decreased by transfection with an miR-374 inhibitor (Fig. 6G).

\section{Discussion}

Many factors are involved in the development of gastric cancer, including high-salt diet, acid secretion, inflammatory cytokines, virulence and colonization of Helicobacter pylori, and host genetic background (14). The establishment of authentic animal models is critical for the prospective study of the pathogenesis of gastric cancer. It is generally believed that gastric stem cells are mainly located in the isthmus of the gastric glands, moving downward or upward to form mature gastric epithelial cells. In our previous study, we reported the isolation of MSCs from human gastric cancer tissues, which provide direct evidence for the presence of MSCs in the gastric cancer microenvironment (15). Whether MSCs are involved in the formation and regulation of the inflammatory microenvironment in gastric cancer was the focus of the present study.
To observe the transformation from gastritis to gastric cancer, we established a gastritis cancer transformation model. Wistar rats were exposed to carcinogen MNNG and fed a high-salt diet which induced the occurrence of gastric cancer.

The histological analyses of the rat stomach tissues at different time points indicated that the gastric mucosa experienced superficial gastritis, atrophic gastritis, intestinal metaplasia and dysplasia, which was similar to the pathological process of human gastric cancer. We detected the expression of several pro-inflammatory factors in the serum of rats collected at different time points using Luminex assay. The expression of MCP-1 was maintained at a high level during the whole process of MNNG induction, which is consistent with our previous study showing that MCP-1 plays an important role in the inflammatory microenvironment (16). In addition, the expression levels of IL-1 $\beta$ and IL-6 markedly increased at the 48th week after MNNG induction, suggesting that IL-1 $\beta$ and IL-6 function at the late stage of malignant transformation. These findings indicate that inflammatory factors are critically involved in the formation of the tumor microenvironment.

We isolated MSCs from the gastric tissues of normal and MNNG-induced rats. RGN-MSCs and RGI-MSCs showed similar stem cell characteristics to that of normal rat bone marrow: mesenchymal cell-like (MSC) morphology, normal karyotype, capabilities of differentiating into osteoblasts and adipocytes, expression of stem cell factors; positive for the mesenchymal cell markers CD29, CD90 and CD44 while negative for the hematopoietic cell surface marker CD45. We further compared the biological characteristics of RGN-MSCs and RGI-MSCs to better understand the effects of the inflammatory microenvironment on MSCs. The proliferation rate of RGI-MSCs was higher than that of the RGN-MSCs due to an increase in the cell population in the $S$ phase. The increased proliferative ability of the RGI-MSCs may reflect an accelerated formation of tumor stroma. RGI-MSCs also had an increased migratory ability than the RGN-MSCs. This finding is consistent with that reported by Park et al who demonstrated that the migratory capacity of MSCs from inflamed periodontal ligament tissues was higher than that from healthy control tissues (17). RGI-MSCs produced higher levels of IL-6, CXCL10 and MCP-1 than RGN-MSCs. The previous study showed that the increased secretion of CCL5 by MSCs resulted in the acceleration of metastatic potential of breast cancer cells (18). Thus, activated MSCs in an inflamed microenvironment may secrete bioactive molecules to promote tumor development and progression.

Epithelial-mesenchymal transition (EMT) is considered to be one of the key steps in tumor initiation, growth and metastasis. Previous studies have demonstrated that direct and indirect interactions with MSCs induce the occurrence of EMT in tumor cells (19-21). We found that RGI-MSCs could induce EMT in gastric epithelial cells more profoundly than RGN-MSCs. Therefore, our findings, along with the findings of previous studies, suggest that MSCs may promote tumor development and progression by the induction of EMT.

We further revealed that the expression of miR-374 was significantly higher in the RGI-MSCs, which positively regulated the proliferation and migration of RGI-MSC. It has been reported that miR-374 promoted the development of gastric cancer $(22,23)$. In addition, miR-374 was highly expressed in 
the tissues or serum of cancer patients (24-26). We previously reported the upregulation of miR-221 in gastric cancer-derived MSCs, which could promote gastric cancer growth and metastasis (15). Therefore, miR-374 may also act as an oncogene in gastric cancer, providing a potential target for the diagnosis and treatment of gastric cancer.

In conclusion, we established a rat model for the transformation of gastritis to gastric cancer using MNNG induction method. MSCs from the inflammatory gastric tissues display increased abilities to proliferate, migrate and produce proinflammatory factors. MSCs from inflammatory gastric tissues were found to have more profound effects in promoting the migration of gastric mucosal epithelial cells. The upregulation of miR-374 may be associated with the phenotypic and functional changes in MSCs in gastric cancer. These findings not only help to better understand the role and mechanism of MSCs in gastric cancer, but also provide new diagnostic and therapeutic targets.

\section{Acknowledgements}

The present study was supported by the Major Research Plan of the National Natural Science Foundation of China (grant no. 91129718), the National Natural Science Foundation of China (grant no. 81572075), the Zhenjiang Science and Technology Program (grant no. SH2016047), the Project of Major Research and Development, Jiangsu Province (grant no. BE2015667), Jiangsu Province for Outstanding Sci-tech Innovation Team in Colleges and Universities (grant no. SJK2013-10), the Doctoral Program Foundation of China (grant nos. 2016M591791 and 2016M591792), the Doctoral Program Foundation, Jiangsu Province (grant no. 1501071C), and the Project Funded by the Priority Academic Program Development of Jiangsu Higher Education Institutions.

\section{References}

1. Norozi F, Ahmadzadeh A, Shahrabi S, Vosoughi T and Saki N: Mesenchymal stem cells as a double-edged sword in suppression or progression of solid tumor cells. Tumour Biol 37: 11679-11689, 2016.

2. Rahmat Z, Jose S, Ramasamy R and Vidyadaran S: Reciprocal interactions of mouse bone marrow-derived mesenchymal stem cells and BV2 microglia after lipopolysaccharide stimulation. Stem Cell Res Ther 4: 12, 2013.

3. Liu Y, Han ZP, Zhang SS, Jing YY, Bu XX, Wang CY, Sun K, Jiang GC, Zhao X, Li R, et al: Effects of inflammatory factors on mesenchymal stem cells and their role in the promotion of tumor angiogenesis in colon cancer. J Biol Chem 286: 25007-25015, 2011.

4. Parkin DM, Bray F, Ferlay J and Pisani P: Global cancer statistics, 2002. CA Cancer J Clin 55: 74-108, 2005.

5. Chen W, Zheng R, Zeng H and Zhang S: The incidence and mortality of major cancers in China, 2012. Chin J Cancer 35: 73, 2016.

6. Yu S, Yang M and Nam KT: Mouse models of gastric carcinogenesis. J Gastric Cancer 14: 67-86, 2014.

7. Hayakawa Y, Fox JG, Gonda T, Worthley DL, Muthupalani S and Wang TC: Mouse models of gastric cancer. Cancers 5: 92-130, 2013 .

8. Zheng MJ, Wang J, Chen YW, Xu L, Xue DD, Fu W, Zhang YF, Du Q, Zhao Y, Ling LJ, et al: A novel mouse model of gastric cancer with human gastric microenvironment. Cancer Lett 325: 108-115, 2012
9. Wang X, Liu H, Wang X, Zeng Z, Xie LQ, Sun ZG and Wei MX: Preventive effect of Actinidia valvata Dunn extract on N-methyl$\mathrm{N}$-nitro-N-nitrosoguanidine-induced gastrointestinal cancer in rats. Asian Pac J Cancer Prev 15: 6363-6367, 2014.

10. Gunassekaran GR, Priya DK, Gayathri R and Sakthisekaran D: In vitro and in vivo studies on antitumor effects of gossypol on human stomach adenocarcinoma (AGS) cell line and MNNG induced experimental gastric cancer. Biochem Biophys Res Commun 411: 661-666, 2011.

11. Bai H, Gu L, Zhou J and Deng D: pl6 hypermethylation during gastric carcinogenesis of Wistar rats by $N$-methyl- $N$ '-nitro- $N$ nitrosoguanidine. Mutat Res 535: 73-78, 2003.

12. Miri H, Bathaie SZ, Mohagheghi MA, Mokhtari-Dizaji M and Shahbazfar AA: A noninvasive method for early detection of MNNG-induced gastric cancer of male Wistar rat: Ultrasonic study. Ultrasound Med Biol 37: 780-787, 2011.

13. Yamabayashi S: Periodic acid-Schiff-alcian blue: A method for the differential staining of glycoproteins. Histochem J 19: 565-571, 1987.

14. Song B, Du J, Feng Y, Gao YJ and Zhao JS: Co-expressed differentially expressed genes and long non-coding RNAs involved in the celecoxib treatment of gastric cancer: An RNA sequencing analysis. Exp Ther Med 12: 2455-2468, 2016.

15. Wang M, Zhao C, Shi H, Zhang B, Zhang L, Zhang X, Wang S, Wu X, Yang T, Huang F, et al: Deregulated microRNAs in gastric cancer tissue-derived mesenchymal stem cells: Novel biomarkers and a mechanism for gastric cancer. Br J Cancer 110: 1199-1210, 2014.

16. Cai J, Wang M, Zhu M, Zhang Q, Zhang X, Yan Y, Qian H and $\mathrm{Xu}$ W: N-methyl-N-nitro-N'-nitrosoguanidine induces the expression of CCR2 in human gastric epithelial cells promoting CCL2-mediated migration. Mol Med Rep 13: 1083-1090, 2016.

17. Park JC, Kim JM, Jung IH, Kim JC, Choi SH, Cho KS and Kim CS: Isolation and characterization of human periodontal ligament (PDL) stem cells (PDLSCs) from the inflamed PDL tissue: In vitro and in vivo evaluations. J Clin Periodontol 38: 721-731, 2011.

18. Melzer C, Yang Y and Hass R: Interaction of MSC with tumor cells. Cell Commun Signal 14: 20, 2016.

19. So KA, Min KJ, Hong JH and Lee JK: Interleukin-6 expression by interactions between gynecologic cancer cells and human mesenchymal stem cells promotes epithelial-mesenchymal transition. Int J Oncol 47: 1451-1459, 2015.

20. Wu C, Zhuang Y, Jiang S, Liu S, Zhou J, Wu J, Teng Y, Xia B, Wang $R$ and Zou X: Interaction between Wnt/ $\beta$-catenin pathway and microRNAs regulates epithelial-mesenchymal transition in gastric cancer (Review). Int J Oncol 48: 2236-2246, 2016.

21. Mele V, Muraro MG, Calabrese D, Pfaff D, Amatruda N, Amicarella F, Kvinlaug B, Bocelli-Tyndall C, Martin I, Resink TJ, et al: Mesenchymal stromal cells induce epithelial-tomesenchymal transition in human colorectal cancer cells through the expression of surface-bound TGF- $\beta$. Int J Cancer 134: 2583-2594, 2014.

22. Xie J, Tan ZH, Tang X, Mo MS, Liu YP, Gan RL, Li Y, Zhang L and Li GQ: MiR-374b-5p suppresses RECK expression and promotes gastric cancer cell invasion and metastasis. World J Gastroenterol 20: 17439-17447, 2014.

23. Xu X, Wang W, Su N, Zhu X, Yao J, Gao W, Hu Z and Sun Y: miR-374a promotes cell proliferation, migration and invasion by targeting SRCIN1 in gastric cancer. FEBS Lett 589: 407-413, 2015.

24. Merhautova J, Hezova R, Poprach A, Kovarikova A, Radova L, Svoboda M, Vyzula R, Demlova R and Slaby O: miR-155 and miR-484 are associated with time to progression in metastatic renal cell carcinoma treated with sunitinib. Biomed Res Int 2015: 941980, 2015

25. Tölle A, Jung M, Rabenhorst S, Kilic E, Jung K and Weikert S: Identification of microRNAs in blood and urine as tumour markers for the detection of urinary bladder cancer. Oncol Rep 30: 1949-1956, 2013.

26. Zhao Q, Li T, Qi J, Liu J and Qin C: The miR-545/374a cluster encoded in the Ftx lncRNA is overexpressed in HBV-related hepatocellular carcinoma and promotes tumorigenesis and tumor progression. PLoS One 9: e109782, 2014. 